\title{
Real-Time Elastography in the diagnosis of prostate cancer: a systematic review
}

\author{
Yang Zhang ${ }^{1 *}$, Zheying Meng ${ }^{1 *}$, Yanjun Xu' ${ }^{1 *}$, Qijie Lu${ }^{1}$, Rui Hou${ }^{1}$, Xiaojun Cai ${ }^{1}$, \\ Lizhou Lin ${ }^{2}$, You Luo ${ }^{3}$, Fengxian Wei ${ }^{4}$, Yuanyi Zheng', Bing Hu' \\ * The authors shared the first authorship
}

\begin{abstract}
${ }^{1}$ Department of Ultrasound in Medicine, Shanghai Institute of Ultrasound in Medicine, Shanghai Jiao Tong University Affliated Sixth People's Hospital, Shanghai, ${ }^{2}$ Department of Ultrasound, Shanghai General Hospital, Shanghai Jiao Tong University School of Medicine, Shanghai, ${ }^{3}$ Department of Urology, the First Affiliated Hospital of Guangdong Pharmaceutical University, Guangzhou, ${ }^{4}$ Department of General Surgery of the Second Hospital of Lanzhou University, Lanzhou, P.R. China
\end{abstract}

\begin{abstract}
Aim: To evaluate the diagnostic accuracy of real-time elastography as a method for detecting prostate cancer. Material and methods: Relevant studies applying real-time elastography as the diagnostic modality and biopsy as the reference standard, published by March 1, 2018 were retrieved from PubMed, EMBASE, Web of Science and Cochrane Library databases. Two independent reviewers inspected all these articles to confirm the matching of the inclusion criteria. One reviewer with methodological expertise extracted the data from the included studies. Sensitivity, specificity and diagnostic odds ratio (DOR) were used to obtain overall estimates. Randomized effect method, meta-regression and subgroup analysis were performed. Results: Twenty-four studies out of 1156 identified articles met the inclusion criteria. Three groups were set: analysis by patient (Group 1), by core (Group 2), and by image (Group 3) and subgroups set in Group 1. The pooled estimate of real-time elastography sensitivity/ specificity/ DOR calculated with the identical P-value 0.00 . Within subgroups "Asia" and "PSA $>=10 \mathrm{ng} / \mathrm{ml}$ ", the pooled sensitivity, specificity and DOR were $0.83,0.65\left(\mathrm{p}=0.01, I^{2}=73.40 \% ; \mathrm{p}=0.02, I^{2}=69.5 \%\right), 0.80$, $0.82\left(\mathrm{p}=0.66, I^{2}=0.00 \% ; \mathrm{p}=0.58, I^{2}=0.00 \%\right)$ and $20.2,8.67\left(\mathrm{p}=0.09, I^{2}=54.2 \% ; \mathrm{p}=0.20, I^{2}=35.5 \%\right)$, respectively. In these three groups, the areas under the SROC curve were $0.7417,0.9246$, and 0.6213 independently. Conclusions: Real-time elastography is a promising, reliable modality for the non-invasive diagnosis of patients with prostate cancer. The diagnostic accuracy of real-time elastography correlates tightly to the presence of higher PSA level and may help avoid unnecessary biopsy. It seems to be a useful tool in systemic biopsy.
\end{abstract}

Keywords: real-time elastography; prostate cancer; diagnosis

\section{Introduction}

In spite of the advances in prostate cancer $(\mathrm{PCa}) \mathrm{de}$ tection and treatment, $\mathrm{PCa}$ continues to be one of the

Received 24.02.2019 Accepted 11.06.2019

Med Ultrason

2019, Vol. 21, No 3, 327-335

Corresponding author: Prof Bing $\mathrm{Hu}$

Department of Ultrasound in Medicine,

Shanghai Institute of Ultrasound in Medicine,

Shanghai Jiao Tong University Affliated Sixth

People's Hospital, 600th Yi Shan Road,

Shanghai 200233, P.R. China

E-mail: binghu_stan@163.com leading causes of cancer-related mortality in men [1], with approximately 180,000 new cases diagnosed and 26,000 cancer-related deaths projected in the United States in 2016 [2]. Hence, the diagnostic accuracy is essential to early intervention regarding tumor progression and metastasis, leading to increasing opportunity for surgery and satisfied prognosis.

Improvement has been achieved through different methods, ranging from digital rectal examination (DRE) to prostate-specific antigen (PSA) levels monitoring, computed tomography (CT) and the most widely-used ultrasonography. Magnetic resonance imaging (MRI), in particular, is an important modality in judging disease 
localization within prostate and an indicator in diagnosis along with the subsequent therapy [3]. As a non-invasive approach, trans-rectal ultrasound (TRUS) is a cost-effective and readily available imaging modality (MRI is not popularized in opportunistic screening due to high cost). Most prostate neoplasms (about 60\%-80\%) are hypoechoic on grey-scale TRUS, whereas $30 \%-40 \%$ are isoechoic, and approximately $1.5 \%$ are hyper-echoic [2]. The sensitivity and specificity of ultrasound for PCa diagnosis vary considerably from $18 \%$ to $96 \%$ and from $46 \%$ to $91 \%$ [1]. Considering the effectiveness and drawbacks of ultrasound, to develop a new protocol is necessary.

Real-time elastography (RTE) is a unique ultrasound (US) mode evaluating physical characteristics of tissue and firstly utilized for targeted prostate biopsies by König in 2005 [4]. The procedure relies on the detection of variance in tissue compliance by manual compression and relaxation and is used in conjunction with grey-scale ultrasound. Nowadays, flexible modalities are well developed: vibration elastography, acoustic radiation force, and shear wave elastography (SWE). The two main types of elastography used in imaging today are compression/ strain and SWE. In the fields of breast, liver and prostate, a series of studies have been reported and meta-analyses were published [5-7].

The aim of the present study was to perform a metaanalysis of published papers to assess the overall accuracy of RTE targeted biopsy in PCa detection based on data analysis: 1) Group 1-by patient, 2) Group 2-by core, and 3) Group 3-by image.

\section{Material and methods}

\section{Search strategy}

The systematic review and meta-analysis were performed according to the Cochrane diagnostic accuracy reviews guidelines. Terms and Medical Subject Headings (MeSH) phrases used included: 'Elasticity Imaging Techniques' [Mesh], elastography AND 'Prostatic Neoplasms' [Mesh], prostate cancer. The search strategy was conducted to find relevant studies from the following databases: Medline (1966 to March 2018), Embase (1980 to March 2018), Cochrane Library (1999 to March 2018), Web of Science (1950 to March 2018).

\section{Study selection}

The inclusion criteria were as follows: 1) diagnostic clinical trials evaluating the accuracy of RTE in the detection of $\mathrm{PCa} ; 2)$ study population: patients with elevated serum PSA ( $>4 \mathrm{ng} / \mathrm{mL})$, or abnormal DRE, or hypo-echoic nodules on TRUS, or low-intensity lesions on T2-weighted images on MRI; 3) use of surgical specimen or TRUS-biopsy as the diagnostic reference standard;
4) English articles; and 5) outcome measurements were in consistency. The exclusion criteria were the following: 1) studies unavailable to construct tables for truepositive, false-positive, false-negative, and true-negative determinations; 2) overlap with the selected studies (ie, studies from the same study group, institution, and period of inclusion); 3) reviews, editorials, case reports, and corresponding letters that did not report their own data; and 4) studies were not suitable for the reference standard.

Two independent reviewers inspected the retrieved studies to identify the conformation with inclusion criteria. Disagreement between two extracting authors was resolved by consensus involving a third party (one of the other members of the research team) after the studies were scrutinized. Values of true positives (TPs), false positives (FPs), false negatives (FNs), and true negatives (TNs) were retrieved from literature. Additional data were extracted from the studies, including first author, publication year, country of origin of the study, study design, number of patients (cores/images), location of biopsy, reference standard for the diagnosis and imaging technique along with the machine used. One reviewer with methodological competence independently extracted the data from the included studies. Any disagreement was resolved by discussion and, if any clarification was necessary, the authors of these studies were contacted.

\section{Statistical methods}

Three groups were set in different aspects: Group 1-by patient, Group 2-by core, and Group 3-by image. Because of significant heterogeneity in Group 1 (analysis by patient), subgroups were made subsequently.

Meta-analysis was performed for the diagnostic accuracy of RTE by calculating pooled estimates of sensitivity, specificity, and diagnostic odds ratio (DOR) in each group. Pooling results (included corresponding 95\% confidence intervals [CIs]) were conducted by using the fixed effect model (Mantel-Haenszel method) when significant heterogeneity was not present; the random-effects model (Der Simonian-Laird method) was applied in which the weights were the inverse of variance of each single study.

The pooled results of sensitivity, specificity, DOR and symmetric receiver operator characteristic (SROC) curve were conducted by using Meta-Disc version 1.4 (Unit of Clinical Biostatistics, Ramony Cajal Hospital, Madrid Spain). The Cochrane Q test was used to detect the heterogeneity among studies. When the $p$ values were $<0.10$ it indicated the presence of heterogeneity. With regard to inconsistency $\left(I^{2}\right)$, the percentage of the variability attributable to heterogeneity, $>50 \%$ was considered significant.

Threshold effect was tested with ROC space and Spearman correlation coefficient [8]. The representation 
of atypical "shoulder arm" pattern in a ROC space and a strong positive correlation between the log of sensitivity and $\log$ of 1 -specificity would suggest presence of threshold effect.

In our study the pooled DORs were obtained calculating the weighted average using the Der-Simonian Laird random effect model. The DORs combined sensitivity and specificity into one measurement of diagnostic performance. The value of a DOR equals to 1 meant the method had no ability to discriminate, while the higher DOR indicated better ability of the test to differentiate subjects with and without the disease of interest. The SROC curve was constructed by using the Moses-Shapiro-Littenberg method to summarize the difference from each study. In addition, the area under the curve (AUC) was calculated, the value ranging from 0.5 to 1.0 . When the value closed to 0.5 indicated a poor test while a value of 1.0 indicated a perfect one.

As for a meta-analysis of diagnostic trials, publication bias can be examined by using Deek's funnel plot. It was conducted by a regression of diagnostic log odds ratio against 1 sqrt (effective sample size [ESS]), weighting by effective sample size, with $P<0.10$ indicating significant asymmetry [9]. Stata (version 12.0) was used for publication bias analysis.

Before performing the statistical analysis, the quality of eligible studies was evaluated with the Quality Assessment of Diagnostic Studies (QUADAS) in Rev-Man (version 5.30). The items of QUADAS were quantified by "yes", "no", or "unclear" with 14 questions [10].

\section{Results}

\section{Eligible studies and quality assessment}

We searched 1156 records and 482 duplicates were removed. After screening titles and abstracts, we identified 104 articles for full-text review. Of these articles, 15 were excluded for type of review articles, 19 for symposiums, 27 for insufficient data, and 19 for different reference standard. Finally, 24 potentially relevant studies were identified as eligible studies [11-34] (fig 1). The quality assessed according to the QUADAS criteria is reported in figure 2.

\section{Study characteristics}

The studies were published between 2002 and 2018, with seven studies performed in Asia. The main characteristics are reported in Table I. Three groups are set in different dimensions: pooled analysis by patient in 16 studies [12,16,17,19,23-34], by core in 9 studies [11-19] and by image in 3 studies [20-22]. In these studies, 2094 patients, 12145 cores and 2182 images were analyzed respectively. Twenty-three studies had a prospective de-

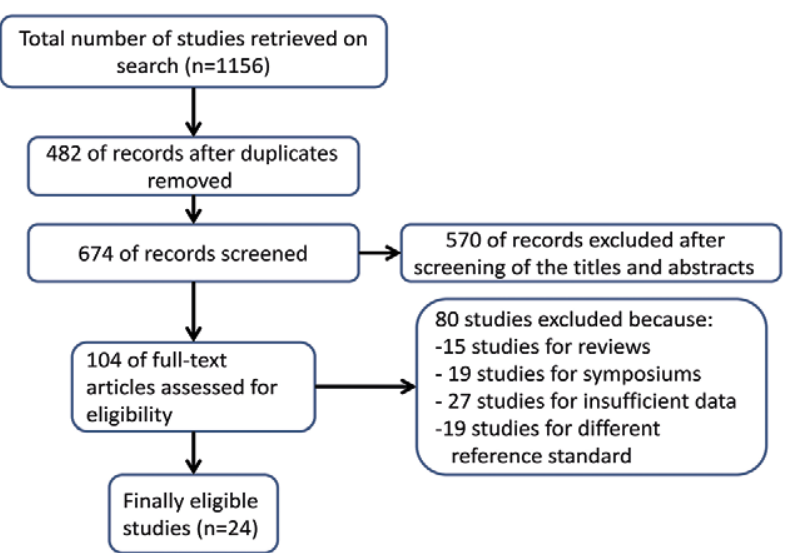

Fig 1. Flowchart of the literature search and selection.
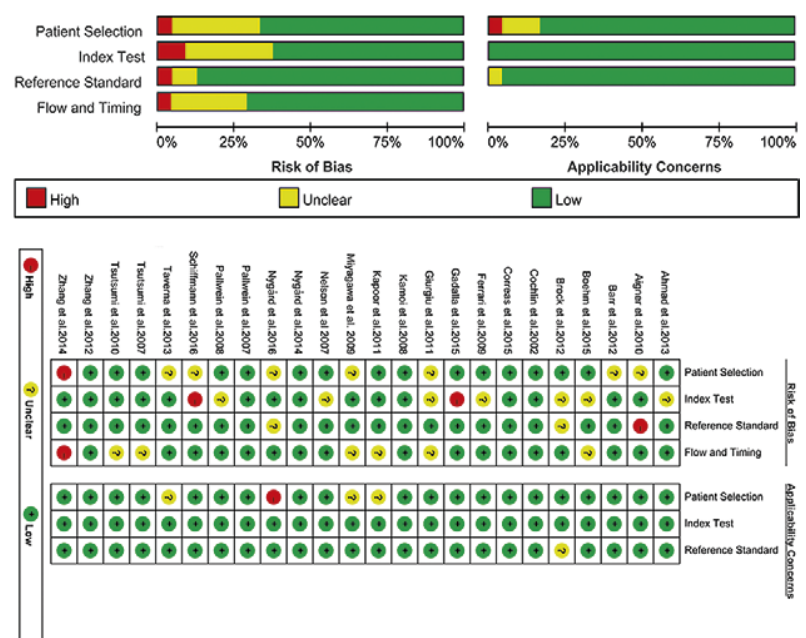

Fig 2. The quality of the eligible studies as assessed according to the Quality Assessment of Diagnostic Accuracy Studies criteria

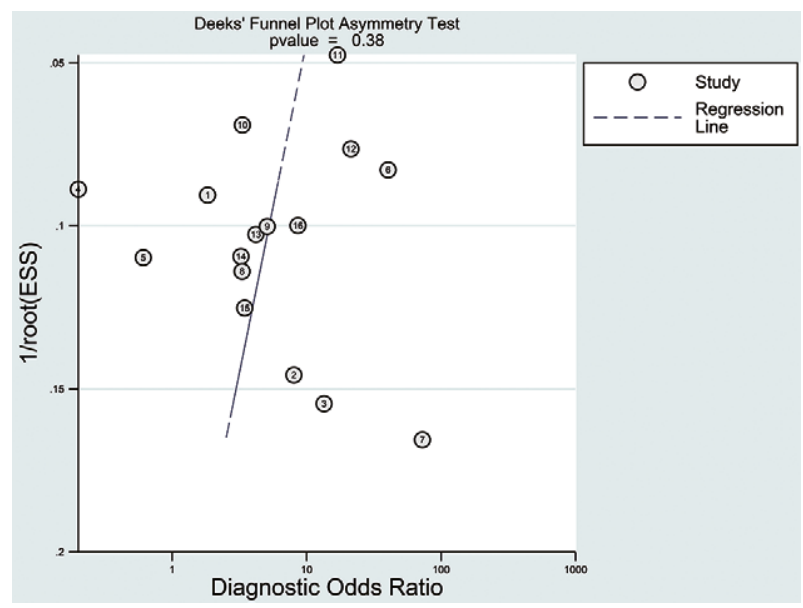

Fig 3. Deek's plot in the test of publication bias $(p=0.38)$. Symmetrical funnel obtained indicated no significant publication bias was introduced in our study. 


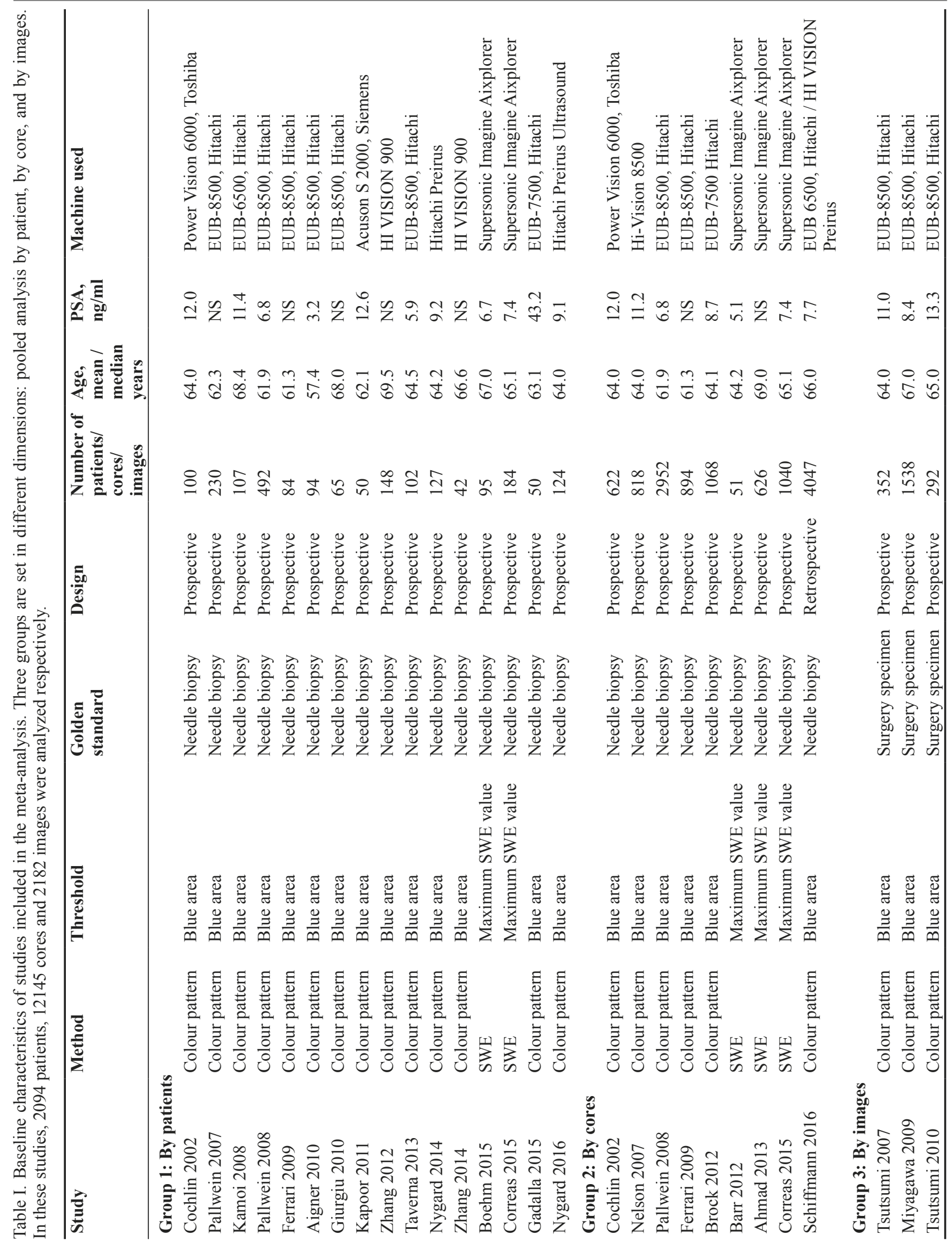


Table II. Results of meta-regression in Group 1 (by patient). "Region" lead to significant heterogeneity. The diagnostic value in "Asia" was 6.91 times higher than "non-Asia" countries $(\mathrm{RDOR}=6.91, \mathrm{p}=0.03)$.

\begin{tabular}{llllll}
\hline Var & Coeff. & Std. Err & $p$-value & RDOR & {$[\mathbf{9 5 \% C I}]$} \\
\hline Cte & 1.090 & 0.4026 & 0.0179 & -- & -- \\
S & 0.155 & 0.2457 & 0.5393 & -- & -- \\
Region & 1.933 & 0.7864 & 0.0288 & 6.91 & $(1.26 ; 37.79)$ \\
\hline
\end{tabular}

sign and fifteen studies clearly stated that the pathologists were blinded to the results of RTE.

Publication bias and influence analysis

Deek's funnel plot (fig 3) showed that $\mathrm{p}=0.38$ ( $>0.10$ ), indicating symmetrical funnel was obtained. It is also observed that the regression line is close to right $90^{\circ}$ vertical to the DOR line. Therefore, no significant publication bias was introduced in our study.

Studies were analyzed after excluding each study in turn and no significant changes are observed in newly pooled sensitivity and specificity, which indicated a good stability of enrolled studies along with credible results generated below.

\section{Pooled analysis by patient (Group 1)}

Significant heterogeneity was found in diagnostic OR $(\mathrm{DOR}=5.06$, Cochrane- $\mathrm{Q}=139.52, \mathrm{df}=15, \mathrm{p}=0.00$, $\left.I^{2}=89.2 \%\right)$. The pooled sensitivity, specificity, positive LR and negative LR and its responding $\mathrm{p}$-value were $0.76(\mathrm{p}=0.00), 0.62(\mathrm{p}=0.00), 2.00(\mathrm{p}=0.00)$, and 0.41 $(\mathrm{p}=0.00)$. Spearman correlation coefficient was 0.032 $(\mathrm{p}=0.91)$, which means no threshold effect existed. Metaregression was performed, and "region" resulted in heterogeneity across the studies. The diagnostic value in "Asia" was 6.91 times higher than in "non-Asia" countries (Table II, RDOR $=6.91, \mathrm{p}=0.03$ ).

Subgroups were set for "region", along with "PSA level", "age", "method", "volume" and "localization ('peripheral zone' or 'whole organ')" of targeted lesions. No significant heterogeneity was found by DOR in the subgroup "Asia" (Table III, DOR=20.2, Cochrane$\left.\mathrm{Q}=6.54, \mathrm{df}=3, \mathrm{p}=0.09, I^{2}=54.2 \%\right)$ and "PSA $>=10 \mathrm{ng} / \mathrm{ml}$ " (DOR=8.67, Cochrane- $\mathrm{Q}=4.65, \mathrm{df}=3, \mathrm{p}=0.20, I^{2}=35.5 \%$ ). The pooled sensitivity in two subgroups were $0.83,0.65$ ( $\left.\mathrm{p}=0.01, I^{2}=73.40 \% ; \mathrm{p}=0.02, I^{2}=69.5 \%\right)$, and specificity $0.80,0.82\left(\mathrm{p}=0.66, I^{2}=0.00 \% ; \mathrm{p}=0.58, I^{2}=0.00 \%\right)$ independently, as shown in figure 4 and 5 .

The SROC curve was obtained. The AUC was 0.7417 (fig 6 - line a).

\section{Pooled analysis by core (Group 2)}

Nine researches were enrolled in this part and there was a heterogeneity. Meta-regression and subgroupsetting were not performed as there were not enough studies in this part. The pooled sensitivity, specificity,

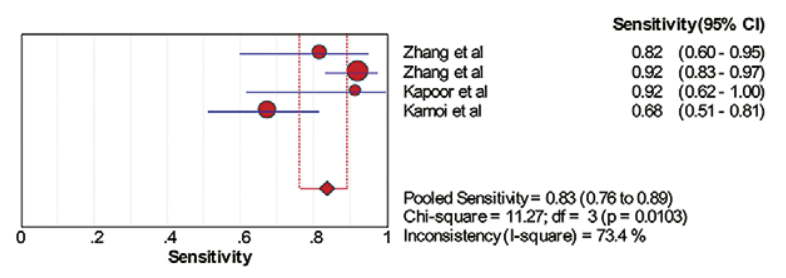

Analysis Options: cells of the studies with zero Filter ON (region $=1$ )
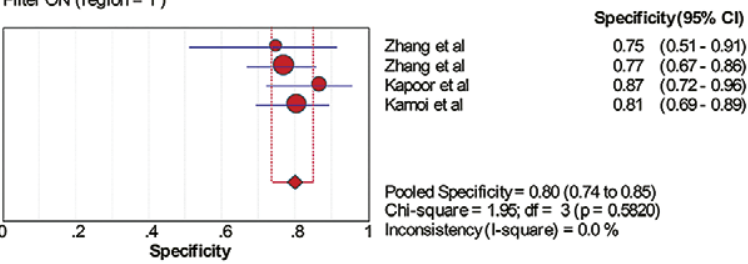

Zhang et al $0.77(0.67-0.86)$ $0.81(0.69-0.89)$

Analysis Options: Filter $\mathrm{ON}(\mathrm{region}=1)$

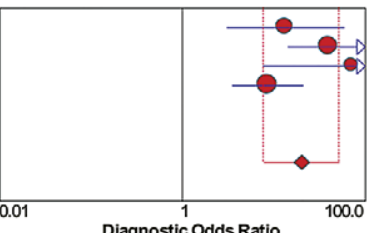

Diagnostic OR $(95 \% \mathrm{Cl})$ Zhang et al Zhang et al Kapoor et a 13.50 (3.07 - 59.46) $40.42(14.20-115.07)$ $72.60(7.63-690.79)$

Random Effects Model Pooled Diagnostic Odds Ratio $=20.23$ (7.78 to 52.64) Cochran- $Q=6.54$; df $=3(p=0.087$

Diagnostic Odds Ratio Tau-squared $=0.4923$ Filder $1 / 2$ to all cells of thegion $=1$ )

Fig 4. Forest plot of subgroup (Asia) of meta-analysis in sensitivity, specificity and DOR for the diagnostic value of RTE in differentiation of prostate cancer. The pooled specificity was $0.80\left(\mathrm{p}=0.66, I^{2}=0.00 \%\right)$.

positive $L R$, negative $L R, D O R$ and their responding $p$ values were $0.51(\mathrm{p}=0.00), 0.88(\mathrm{p}=0.00), 4.30(\mathrm{p}=0.00)$, $0.41(\mathrm{p}=0.00)$, and $11.76(\mathrm{p}=0.00)$. The SROC curve was drawn. The AUC was 0.9246 (fig 6-line b).

\section{Pooled analysis by image (Group 3)}

A heterogeneity was found. The pooled sensitivity, specificity, positive LR, negative LR, DOR and their responding values were $0.77(\mathrm{p}=0.00), 0.36(\mathrm{p}=0.00), 1.22$ $(\mathrm{p}=0.00), 0.52(\mathrm{p}=0.00)$, and $2.38(\mathrm{p}=0.00)$. The AUC was 0.6213 (fig 6-line c).

\section{Discussions}

Screening, detection and diagnosis of prostate cancer are currently on the basis of DRE, PSA levels, CT, MRI and greyscale ultrasound. However, limitations to these approaches in clinical practice are clear. Digital examination depends mainly on the experience of the physician subjectively and, occult lesions or minor ones cannot be detected effectively; PSA levels can be influenced by infections, hyperplasia, drugs in-taken and so on, following a poor specificity and low predictive value; $\mathrm{CT}$ virtually 


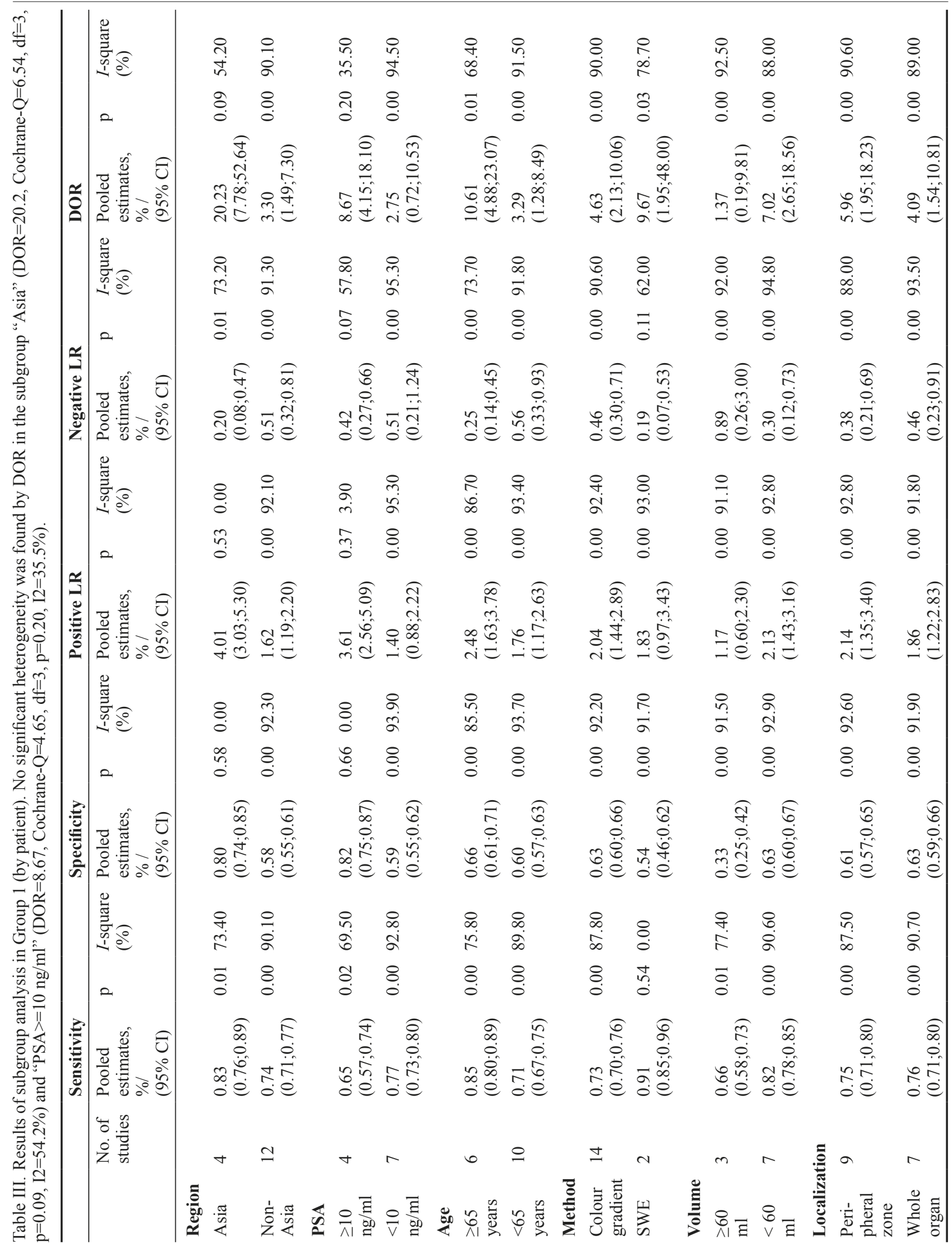


plays no role in the detection of $\mathrm{PCa}$ and is not recommended for diagnostic purposes as soft tissues resolution is low and margins of the gland are poorly defined [1]. Conventional MRI is of value in predicting the pathological stage and extra-capsular extension. Prostate MRI used before first prostate biopsy, could identify men who might safely avoid an unnecessary biopsy. However, it is less cost-effective in active surveillance. The use of TRUS has limited diagnostic accuracy, for benign lesions such as prostatitis, infarction, and benign prostate hypertrophy, may be hypo-echoic, resulting to varying sensitivity and specificity.

Within the stages including the occurrence and progression of neoplasms, cancerous lesions became harder and less elastic, following the changes histologically: increased cellular density, rising micro-vascularity, a loss of glandular tissue architecture [35], and increased collagen deposition in the stroma [36]. Real-time elastography is applied in detecting abnormal lesions by images accessing of tissue elasticity or stiffness, involving many fields like thyroid, liver, kidney, breast, pancreas and prostate [37-43]. As a modality of great importance, however, the sensitivity and specificity are variously reported. Our study was designed to analyze pooled data and evaluate the diagnostic value of RTE in the detection of prostate cancer.

Though meta-analyses have been performed before $[6,7,43]$, updates based on new and large-scale data is still necessary, with setting of subgroups and deeper exploration. As an invasive procedure, TRUS-guided biopsy may cause infections and hematuria. Over the past decade, there has been a trend to obtain larger numbers of biopsy specimens, with most clinicians taking 8 to 12 biopsy cores and most current studies recommending a 12-core biopsy scheme [44]. According to our study, real-time elastography will decrease the rate of unnecessarily performed cores with lower health care costs and side effects.

Subgroups were set in Group 1 by considering region, PSA levels, age, prostate volume, method and tumor localization based on the results of meta-regression and clinical perspectives. Total serum PSA, prostate volume and age have been found to be clinically significant predictors of positive biopsy findings [45]. Within the subgroup of PSA level $>=10 \mathrm{ng} / \mathrm{ml}$ and region of Asia, the pooled sensitivity was $0.65,0.83$ and specificity 0.82 , 0.80 . No significant heterogeneity was found in specificity ( $\left.\mathrm{p}=0.66, I^{2}=0.00 \% ; \mathrm{p}=0.58, I^{2}=0.00 \%\right)$. This indicated that patients in Asia counties and/or with higher PSA may be more likely to be precisely diagnosed by RTE. The values of RTE climbed as the PSA level increased, meaning the progression of tumor, and that RTE is a reliable indicator in the detection of abnormal lesions. As to
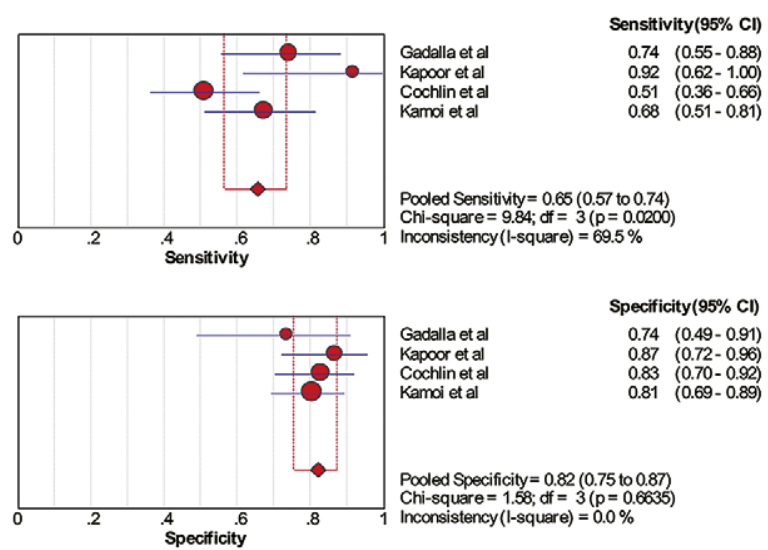

Gadalla et al $0.87(0.72-0.96$ Kamoi et al $\quad 0.81(0.69-0.89)$
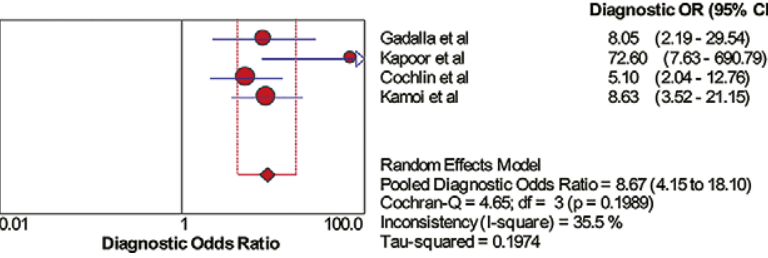

Fig 5. Forest plot of subgroup (PSA $>=10 \mathrm{ng} / \mathrm{ml}$ ) of meta-analysis in sensitivity, specificity and DOR for the diagnostic value of RTE in differentiation of prostate cancer. The pooled specificity was $0.82\left(\mathrm{p}=0.58, I^{2}=0.00 \%\right)$.

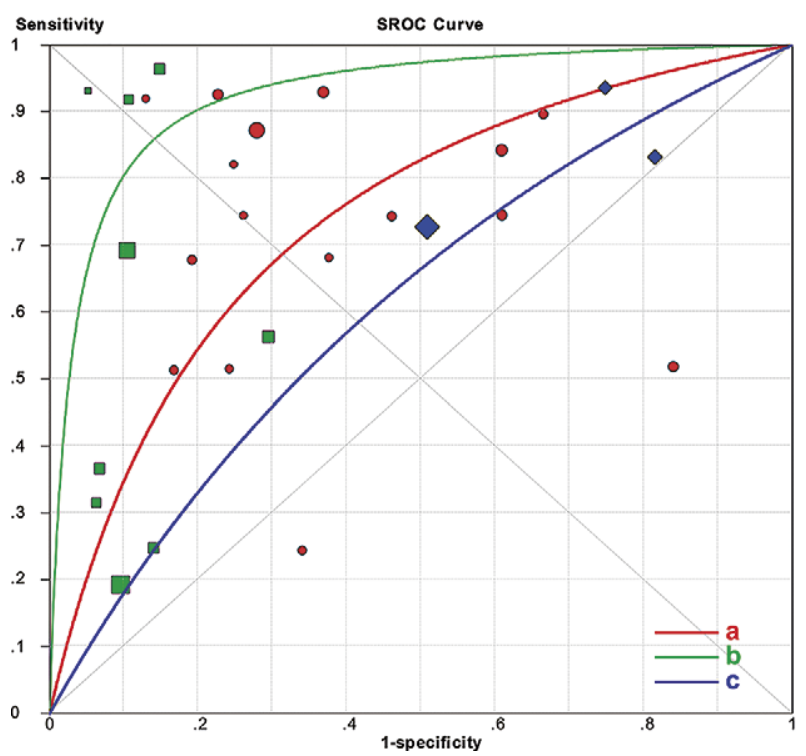

Fig 6. The summary receiver operating characteristics (SROC) curve for the diagnostic value of RTE in differentiation of prostate cancer. Line a (red), b (green) and c (blue) referred to the analysis by patient, by core and by image. The AUC was $0.7417,0.9246,0.6213$ literally.

the data in Asian areas, the diagnostic odds ratio is six times higher than that in non-Asian countries, but with a $I$-square $(54.2 \%)$ which indicated the inconsistency 
within this group. Not many relevant studies (only 4) that conform with our standards were well performed in Asia, and this is the principal reason leading to the high DOR in this subgroup. More studies and following updates are still required.

To our knowledge, this is the first meta-analysis introducing analysis of RTE with three dimensions: by patient, by core and by image respectively, along with both reference standard of TRUS-guided biopsy and radicalprostatectomy biopsy. According to the results of our study, the diagnostic accuracy of RTE quantified by AUC is 0.9246 for the diagnosis of prostate cancer in the group "analysis by core". Our meta-analysis indicated that RTE can be used with a high diagnostic accuracy to detect malignant prostate lesions.

There are several limitations to our study. First, only three studies were included in the analysis by image. Second, though high sensitivity, specificity and DOR are obtained with fine consistency in subgroup "Asia" and "PSA $>=10 \mathrm{ng} / \mathrm{ml}$ ", the small number of studies as a factor cannot be neglected which will give rise to higher estimates. Third, as data on Gleason score were not well described in research the analysis was not performed unfortunately. Fourth, RTE is a modality dependent on physicians' performance. Though colour-gradient scale was applied in ultrasound system, the judgment of the right images and localization is still influenced by the pressure given by doctors and their experience. Reproducible results cannot be obtained satisfactorily. Modalities like SWE which uses acoustic radiation force and are less dependent on performers need to be popularized. In addition, in this paper, the publication bias cannot be excluded, even though the analysis of the Deek's plot indicated small risk.

In conclusion, in our study, RTE achieved an exciting efficiency in PCa detection, with the analysis of sensitivity, specificity and diagnostic odds ratio. Furthermore, it showed quite correspondence with the PSA elevation, indicating its value as a potential marker. Due to the obtained results and to their non-invasive characteristics, RTE is a promising modality for the early characterization in patients with prostate cancer and may reduce the unnecessary biopsies in the future, along with digital rectal examination, serum indicator, greyscale US and MRI. Large-scale trials are warranted to further explore the diagnostic value of RTE in the diagnosis of prostate cancer and other fields.

Acknowledgement: This study was supported by the Shanghai Key Discipline of Medical Imaging (No: 2017ZZ02005), Clinical Science and Technology Innovation Project (grant nos. SHDC22015001) and the Natural Science Foundation of China (grant nos. 81271597, 81401421 and 81372732).

\section{Conflict of interest: none}

\section{References}

1. Pummer K, Rieken M, Augustin H, Gutschi T, Shariat SF. Innovations in diagnostic imaging of localized prostate cancer. World J Urol 2014;32:881-890.

2. Chen FK, de Castro Abreu AL, Palmer SL. Utility of Ultrasound in the Diagnosis, Treatment, and Follow-up of Prostate Cancer: State of the Art. J Nucl Med 2016;57 (Suppl 3):13S-18S.

3. Kuru TH, Fütterer JJ, Schiffmann J, Porres D, Salomon G, Rastinehad AR. Transrectal Ultrasound (US), Contrastenhanced US, Real-time Elastography, HistoScanning, Magnetic Resonance Imaging (MRI), and MRI-US Fusion Biopsy in the Diagnosis of Prostate Cancer. Eur Urol Focus 2015;1:117-126.

4. König K, Scheipers U, Pesavento A, Lorenz A, Ermert H, Senge T. Initial experiences with real-time elastography guided biopsies of the prostate. J Urol 2005;174:115-117.

5. Ouyang Q, Duan Z, Lei J, Jiao G. Comparison of metaanalyses among elastosonography (ES) and positron emission tomography/computed tomography (PET/CT) imaging techniques in the application of prostate cancer diagnosis. Tumor Biol 2016;37:2999-3007.

6. Teng J, Chen M, Gao Y, Yao Y, Chen L, Xu D. Transrectal sonoelastography in the detection of prostate cancers: a meta-analysis. BJU Int 2012;110:E614-E620.

7. Zhang B, Ma X, Zhan W, et al. Real-time elastography in the diagnosis of patients suspected of having prostate cancer: A meta-analysis. Ultrasound Med Biol 2014;40:1400-1407.

8. Dinnes J, Deeks J, Kirby J, Roderick P. A methodological review of how heterogeneity has been examined in systematic reviews of diagnostic test accuracy. Health Technol Assess 2005;9:1-113.

9. Deeks JJ, Macaskill P, Irwig L. The performance of tests of publication bias and other sample size effects in systematic reviews of diagnostic test accuracy was assessed. J Clin Epidemiol 2005;58:882-893.

10. Whiting P, Rutjes AW, Reitsma JB, Bossuyt PM, Kleijnen J. The development of QUADAS: a tool for the quality assessment of studies of diagnostic accuracy included in systematic reviews. BMC Med Res Methodol 2003;3:25.

11. Schiffmann J, Grindei M, Tian Z, et al. Limitations of Elastography Based Prostate Biopsy. J Urol 2016;195:17311736.

12. Correas JM, Tissier AM, Khairoune A, et al. Prostate cancer: diagnostic performance of real-time shear-wave elastography. Radiology 2015;275:280-289.

13. Ahmad S, Cao R, Varghese T, Bidaut L, Nabi G. Transrectal quantitative shear wave elastography in the detection and characterisation of prostate cancer. Surg Endosc 2013;27:3280-3287.

14. Brock M, Von Bodman C, Palisaar RJ, et al. The impact of real-time elastography guiding a systematic prostate biopsy to improve cancer detection rate: A prospective study of 353 patients. J Urol 2012;187:2039-2043. 
15. Barr RG, Memo R, Schaub CR. Shear wave ultrasound elastography of the prostate: Initial results. Ultrasound Q 2012;28:13-20.

16. Ferrari FS, Scorzelli A, Megliola A, Drudi FM, Trovarelli S, Ponchietti R. Real-time elastography in the diagnosis of prostate tumor. J Ultrasound 2009;12:22-31.

17. Pallwein L, Mitterberger M, Pinggera G, et al. Sonoelastography of the prostate: Comparison with systematic biopsy findings in 492 patients. Eur J Radiol 2008;65:304-310.

18. Nelson ED, Slotoroff CB, Gomella LG, Halpern EJ. Targeted biopsy of the prostate: the impact of color Doppler imaging and elastography on prostate cancer detection and Gleason score. Urology 2007;70:1136-1140.

19. Cochlin DL, Ganatra RH, Griffiths DFR. Elastography in the detectionofprostaticcancer.ClinRadiol2002;57:1014-1020.

20. Tsutsumi M, Miyagawa T, Matsumura T, et al. Real-time balloon inflation elastography for prostate cancer detection and initial evaluation of clinicopathologic analysis. AJR Am J Roentgenol 2010;194:W471-476.

21. Miyagawa T, Tsutsumi M, Matsumura T, et al. Real-time elastography for the diagnosis of prostate cancer: Evaluation of elastographic moving images. Jpn J Clin Oncol 2009;39:394-398.

22. Tsutsumi M, Miyagawa T, Matsumura T, et al. The impact of real-time tissue elasticity imaging (elastography) on the detection of prostate cancer: clinicopathological analysis. Int J Clin Oncol 2007; 12:250-255.

23. Nygård Y, Haukaas SA, Halvorsen OJ, et al. A positive Real-Time Elastography (RTE) combined with a Prostate Cancer Gene 3 (PCA3) score above 35 convey a high probability of intermediate- or high-risk prostate cancer in patient admitted for primary prostate biopsy. BMC Urology 2016;16:39.

24. Gadalla AAEFH, El Rahman SFA, Anis SE, Khalil MES. Value of ultrasound elastography versus transrectal prostatic biopsy in prostatic cancer detection. Egypt J Radiol Nucl Med 2015;46:761-768.

25. Boehm K, Budäus L, Tennstedt P, et al. Prediction of Significant Prostate Cancer at Prostate Biopsy and per Core Detection Rate of Targeted and Systematic Biopsies Using RealTime Shear Wave Elastography. Urol Int 2015;95:189-196.

26. Zhang Y, Tang J, Liang HD, Lv FQ, Song ZG. Transrectal Real-time Tissue Elastography - An Effective Way to Distinguish Benign and Malignant Prostate Tumors. Asian Pac J Cancer Prev 2014;15:1831-1835.

27. Nygård Y, Haukaas SA, Halvorsen OJ, et al. A positive realtime elastography is an independent marker for detection of high-risk prostate cancers in the primary biopsy setting. BJU Int 2014;113:E90-E97.

28. Taverna G, Magnoni P, Giusti G, et al. Impact of real-time elastography versus systematic prostate biopsy method on cancer detection rate in men with a serum prostatespecific antigen between 2.5 and $10 \mathrm{ng} / \mathrm{mL}$. ISRN Oncol 2013;2013:584672.

29. Zhang Y, Tang J, Li YM, et al. The contribution of strain patterns in characterization of prostate peripheral zone lesions at transrectal ultrasonography. Acta Radiol 2012;53:119-126.
30. Kapoor A, Kapoor A, Mahajan G, Sidhu BS. Real-Time Elastography in the Detection of Prostate Cancer in Patients with Raised PSA Level. Ultrasound Med Biol 2011;37:1374-1381.

31. Giurgiu CR, Manea C, Crişan N, Bungărdean C, Coman I, Dudea SM. Real-time sonoelastography in the diagnosis of prostate cancer. Med Ultras 2011;13:5-9.

32. Aigner F, Pallwein L, Junker D, et al. Value of real-time elastography targeted biopsy for prostate cancer detection in men with prostate specific antigen $1.25 \mathrm{ng} / \mathrm{ml}$ or greater and $4.00 \mathrm{ng} / \mathrm{ml}$ or less. J Urol 2010;184:913-917.

33. Kamoi K, Okihara K, Ochiai A, et al. The utility of transrectal real-time elastography in the diagnosis of prostate cancer. Ultrasound Med Biol 2008;34:1025-1032.

34. Pallwein L, Mitterberger M, Struve P, et al. Comparison of sonoelastography guided biopsy with systematic biopsy: Impact on prostate cancer detection. Eur Radiol 2007; 17:2278-2285.

35. Good DW, Stewart GD, Hammer S, et al. Elasticity as a biomarker for prostate cancer: a systematic review. BJU Int 2014;113:523-534.

36. Tuxhorn JA, Ayala GE, Smith MJ, Smith VC, Dang TD, Rowley DR. Reactive stroma in human prostate cancer: induction of myofibroblast phenotype and extracellular matrix remodeling. Clin Cancer Res 2002;8:2912-2923.

37. Yang Z, Zhang H, Wang K, Cui G, Fu F. Assessment of diffuse thyroid disease by strain ratio in ultrasound elastography. Ultrasound Med Biol 2015;41:2884-2889.

38. Abo Elwafa W. Role of elastography in diagnosis of suspecious thyroid nodules. Eur Thyroid J 2013;2:118.

39. Conti CB, Cavalcoli F, Fraquelli M, Conte D, Massironi S. Ultrasound elastographic techniques in focal liver lesions. World J Gastroenterol 2016;22:2647-2656.

40. Anvari A, Barr RG, Dhyani M, Samir AE. Clinical application of sonoelastography in thyroid, prostate, kidney, pancreas, and deep venous thrombosis. AbdomImaging 2015;40:709-722.

41. Zhi H, Xiao XY, Yang HY, Ou B, Wen YL, Luo BM. Ultrasonic Elastography in Breast Cancer Diagnosis: Strain Ratio vs 5-point Scale. Acad Radiol 2010;17:1227-1233.

42. Wang R, Chen JJ, Hu B. Transrectal real-time elastographyguided transperineal prostate biopsy as an improved tool for prostate cancer diagnosis. Int J Clin Exp Med 2015;8:65226529.

43. Aboumarzouk O, Ogston S, Huang Z, et al. Diagnostic accuracy of transrectal elastosonography (TRES) imaging for the diagnosis of prostate cancer: a systematic review and meta-analysis. BJU Int 2012;110:1414-1423.

44. Hwang SI, LeeHJ. The future perspectives in transrectal prostate ultrasound guided biopsy. Prostate Int 2014;2:153-160.

45. Murray NP, Reyes E, Fuentealba C, Orellana N, Morales F, Jacob O. Comparison of the Formula of PSA, Age, Prostate Volume and Race Versus PSA Density and the Detection of Primary Malignant Circulating Prostate Cells in Predicting a Positive Initial Prostate Biopsy in Chilean Men with Suspicion of Prostate Cancer. Asian Pac J Cancer Prev 2015;16:5365-5370 